\title{
Efectividad de especies naturales como ayudantes de Coagulación, para la clarificación de aguas turbias en épocas de avenidas en caseríos y centros poblados de Huaraz y Callejón de Huaylas
}

Effectiveness of natural species Coagulation as assistants for clarification of turbid water in times of flood in villages and towns of Huaraz and Callejon de Huaylas

\author{
Cesar Manuel Dávila Paredes ${ }^{1}$, Martín Miguel Huamán Carranza ${ }^{1}$, Judith Isabel \\ Flores Albornoz ${ }^{1}$, Rosario Adriana Polo Salazar ${ }^{1}$ Y Nino Franklin Araujo Jamanca ${ }^{1}$
}

\section{RESUMEN}

Las poblaciones que consumen agua de fuentes hídricas deben garantizar una buena calidad para su consumo, por ello se tuvo por objetivo evaluar la efectividad de especies naturales como ayudantes de coagulación para la clarificación de aguas turbias que se generan en épocas de avenidas en los ríos LLullan, Paria y Chucchun. La metodología aplicada: Recolección de los coagulantes, Test de jarras y determinación de dosis optimas y pH. Se utilizó coagulantes naturales como la mashua, papa, tara, penca y trigo, que se añadieron a la muestra en el ensayo de test de jarras, obteniéndose una dosis de $45 \mathrm{mg} / \mathrm{l}$ a un $\mathrm{pH}$ de agua de 7,5 con concentraciones de 5,3\% obteniéndose valores de remoción importantes para los parámetros de turbiedad y solidos disueltos totales. Al evaluar la eficiencia se comprobó que existe remoción de turbiedad por debajo de la norma, con valores de eficiencia de $89,9 \%$ para los coagulantes de mashua, papa, tara, penca y trigo, los coagulantes alcanzaron la remoción de solidos disueltos de hasta el $89 \%$ en la tara y un mínimo de $13,5 \%$ en el trigo valor exigido por la norma. Por tanto, es posible utilizar insumos naturales como coagulantes para la clarificación de aguas superficiales en épocas de lluvias y pode potabilizarlas y ser aptas para consumo humano.

Palabras clave: coagulantes; turbiedad; potabilizar; eficiencia; clarificación.

1 Universidad Nacional Santiago Antúnez de Mayolo. Huaraz, Perú.

(C) Los autores. Este artículo es publicado por la Revista Aporte Santiaguino de la Universidad Nacional Santiago Antúnez de Mayolo. Este es un artículo de acceso abierto, distribuido bajo los términos de la Licencia Creative Commons Atribución-NoComercial-CompartirIgual 4.0 Internacional. (http://creativecommons.org/licenses/ by-nc-sa/4.0/), que permite el uso no comercial, distribución y reproducción en cualquier medio, siempre que la obra original sea debidamente citada. 


\begin{abstract}
The populations that consume water from water sources must guarantee a good quality for their consumption, for that reason the objective was to evaluate the effectiveness of natural species as coagulation aids for the clarification of turbid waters that are generated in times of floods in the LLullan rivers, Paria and Chucchun. The applied methodology: Collection of coagulants, jar test and determination of optimal doses and $\mathrm{pH}$. Natural coagulants such as mashua, potato, tara, penca and wheat were used, which were added to the sample in the jar test, obtaining a dose of $45 \mathrm{mg} / \mathrm{l}$ at a water $\mathrm{pH}$ of 7,5 with concentrations of $5,3 \%$, obtaining important removal values for turbidity parameters and total dissolved solids. When evaluating the efficiency, it was verified that there is removal of turbidity below the norm, with efficiency values of $89,9 \%$ for mashua, potato, tara, penca and wheat coagulants, the coagulants reached the removal of dissolved solids up to $89 \%$ in the tare and a minimum of $13,5 \%$ in the wheat value required by the standard. Therefore, it is possible to use natural inputs as coagulants for the clarification of surface waters during rainy seasons and to make them drinkable and suitable for human consumption.
\end{abstract}

Keywords: coagulants; turbidity; purification; efficiency.

\title{
INTRODUCCIÓN
}

La investigación tiene por objetivo proponer formas de clarificación de agua para las zonas urbanas y rurales cuyas aguas se ven afectadas por fenómenos naturales, como la crecida de los ríos en épocas de avenidas y desastres naturales, la falta de conocimiento de tecnologías sencillas para la potabilización del agua hace que la investigación tenga la importancia ambiental, sostenible y económica. El tema es relevante porque en las zonas urbanas y rurales de Huaraz captan sus aguas de fuentes de manantial y canales superficiales, para consumo humano y en épocas de lluvia o desastres naturales existen alteraciones de la calidad del agua, debido a que no cuentan con un tratamiento adecuado las aguas se vuelven turbias por tanto no son aceptables para consumo. Se sabe que, si se emplea un coagulante químico más los insumos para el proceso de desinfección, la operación y mantenimiento resulta costoso, como no existe una adecuada educación de pago por el servicio y una mala gestión del personal encargado de la operación y mantenimiento, en la actualidad son manejados por las juntas administrativas de agua potable de las diferentes municipalidades. En el sector rural de la ciudad de Huaraz es obligatorio revertir esta situación ya que el consumo de agua sin tratamiento y potabilización genera problemas en la salud de la población. El uso de un producto natural en la zona que tenga propiedades de las sales de aluminio y fierro para clarificar el agua, empleando métodos básicos y capacitación adecuada al personal encargado, se puede 
mejorar el tratamiento del agua y disminuir los costos en los procesos de coagulación, floculación y filtración, considerándose económicos a comparación de los equipos patentados.

La investigación tiene como objetivos evaluar la calidad de agua, determinar la cantidad, dosis optima, concentración de las especies naturales como ayudantes de coagulación para la clarificación. Además, determinar la eficiencia de las especies naturales como coagulante natural en la clarificación de agua para consumo humano y finalmente determinar el insumo de mejor eficiencia y disponibilidad como alternativa de uso en la potabilización del agua en zona rural de Huaraz y Callejón de Huaylas.

Yunusa U. (2017) en su trabajo denominado "Cucurbita pepo Seed Powder Reduce the Turbidity of River Water" manifiesta "El alto costo del agua tratada hace que la mayoría de las personas en las comunidades rurales recurran a fuentes de agua que son normalmente de baja calidad y que los exponen a enfermedades transmitidas por el agua. El principal objetivo de este trabajo es evaluar la efectividad de los polvos de semilla de pepino Cucurbita pepo para la eliminación de la turbidez en el agua del río utilizando Alum como un estándar. Se recogieron muestras de agua del río Gara, área del gobierno local de Madobi, estado de Kano, Nigeria.

Se prepararon diversas concentraciones de polvo de semillas de Alum y C. pepo; 100, 200, 300 y 400 ppm. pH, turbidez, sólidos disueltos totales, conductividad eléctrica, sólidos suspendidos totales y temperatura de las muestras fueron analizado antes y después del tratamiento. Se observó que los polvos de semilla de C. pepo disminuyen el TDS mientras que el alumbre se observa un aumento de TDS. Este estudio indica que los polvos de semilla de C. pepo podrían proporcionar una turbidez baja y una alternativa por su capacidad de reducción en el agua del río, por su disponibilidad y bajo costo". Pavanakumar P., Venkatareddy K. y Keerthi G. (2017) en su trabajo de investigación denominado "Application of Natural Coagulants in Removal of Turbidity and Hardness", manifiestan "La turbidez en el agua es causada por la presencia de arcillas, sedimentos, partículas orgánicas e inorgánicas finamente divididas y la dureza es causada por la presencia de un exceso de minerales, sustancia soluble, etc. La eliminación de la turbidez y la dureza es uno de los pasos importantes en el proceso de tratamiento del agua. Se logra mediante el proceso de coagulación utilizando coagulantes de base química. La eficacia del coagulante químico está bien notada, pero no se pueden descartar los inconvenientes asociados con ella, como su alto costo, los efectos perjudiciales para la salud humana y la gran producción de lodos. Por lo tanto, es necesario reemplazar el coagulante químico con coagulantes naturales rentables. Cactus Opuntia fue eficaz para el agua turbia alta con una eliminación de la turbidez del 99\%, mientras que la eliminación de la turbidez fue del 94\% y 83\% para el agua turbia media y baja, respectivamente. Consolea Rubescens mostró una reducción porcentual máxima correspondiente al 
97\%, 93\% y 95\% para aguas turbias bajas, medias y altas, respectivamente. Moringa Oleifera tiene la capacidad de eliminar la dureza máxima a medida que aumenta la dosis".

Kumar (2015) en su trabajo de investigación "Coagulation performance evaluation of natural and synthetic coagulants in waste water treatment" manifiesta "El método actual utiliza varios productos químicos que sintetizan significativamente los subproductos que pueden contaminar el medio ambiente y puede deteriorar el ecosistema a un ritmo lento. Para que el proceso de coagulación sea ecológico y eficiente. En este estudio se intenta evaluar el Rendimiento de la coagulación de algunos materiales naturales y sintéticos para eliminar las partículas suspendidas en las aguas residuales. Los Eliminación de partículas en suspensión en función del tiempo, la dosis y la turbidez inicial. El intento de turbidez, la eliminación seguida por la eliminación de Sólidos Disueltos Totales (TDS) ha sido prevista, la eliminación de TDS por continuo, las técnicas de columna de flujo se planean utilizando arena mixta de TiO2. Los efluentes de la industria textil y de aguas residuales se planean para ser tratados mediante la adopción de las técnicas de eliminación anteriores. El coagulante sintético con el que tuvimos una mayor coagulación, eficiencia y se puede utilizar para tratar efluentes de turbidez más alta".

Rodiño (2015) manifestó "Se evaluaron cinco extractos coagulantes naturales en solución salina de tallos de Hylocereus cf. Trigonus(Cactus), exudado gomoso de Albizia saman(Campano), corteza de Guazuma ulmifolia(Guácimo) y corteza y semilla de Moringa oleiffera(Moringa), en muestras de agua cruda tomadas del río Sinú, con niveles de turbidez inicial entre 56 y 300 unidades nefelométricas de turbidez (NTU). Con ensayos de jarras, se determinó las eficiencias de remoción de turbidez, como una función del porcentaje de actividad coagulante, para dosis aplicadas entre $5 \mathrm{mg} / \mathrm{L}$ a $200 \mathrm{mg} / \mathrm{L}$. Aunque el Carbono Orgánico Total (COT) es un parámetro importante en la calidad del agua, no se incluyó en este estudio debido a que se ha hallado que la turbidez del rio Sinú es de origen sedimentario y su corriente tiene una baja carga orgánica. Las eficiencias de remoción variaron de 40\% (con turbidez menores a $100 \mathrm{NTU}$ ) hasta $90 \%$ (con turbidez inicial mayor a $150 \mathrm{NTU}$ ), para dosis óptimas de $10 \mathrm{mg} / \mathrm{L}$ a $40 \mathrm{mg} / \mathrm{L}$ de extractos de tallos de $H$. cf. trigonus, exudado gomoso de $A$. saman, y cortezas de $G$. ulmifolia y de $M$. oleifera. El extracto de mayor eficiencia fue el obtenido de la semilla de M. oleifera, siendo más efectivo con turbidez mayor a 150 NTU, logrando una actividad coagulante hasta del 98\%".

Aguilar (2010) plantea que "La utilización de polímeros naturales es una interesante alternativa por su bajo costo en comparación con los polímeros sintéticos, en el caso de este producto es totalmente natural e inocuo para las personas. La goma de tara se produce en el Perú en grandes cantidades, para después pasar por una filtración básica o convencional y seguida de una unidad de desinfección". 
La presente investigación pretende dar un aporte para proponer alternativas sencillas y de fácil manejo por las personas en caso de cualquier eventualidad, como en los fenómenos naturales que ocasionan problemas y daños en los servicios básicos de abastecimiento de agua potable de las comunidades más vulnerables del callejón de Huaylas. Se conoce que en Huaraz y el Callejón de Huaylas el crecimiento natural de la familia de Agave Americano (Penca), Triticum Vulgare (Trigo), Prunus Persica (Melocotón), Aloe Vera (Sábila), Echinopsis peruviana (Cactus San Pedro), Almidon de Manihot Esculenta (Yuca), Caesalpinia Spinosa (Tara), Vicia Faba (Habas), Tropaeolum Tuberosum (Mashua), Solanum Tuberosum (Cascara de Papa), Almidón de Zea Mays (Maíz), Opuntia Ficus - Indica (Tuna); entre otros géneros y especies, por estudios realizados en diferentes partes del país el empleo de coagulantes naturales es beneficioso para la purificación de aguas para consumo como para aguas residuales.

El trabajo se justifica en demostrar el empleo eficiente de coagulantes naturales como ayudantes de coagulación y que sean útiles de emplear fenómenos de lluvias intensas de alta generación de turbiedad y por condiciones de variación debido al cambio climático, promoviendo otro insumo para la coagulación de origen natural que evita a largo plazo el gasto en insumos químicos y la acumulación de estos en la salud de las personas y plantear métodos sencillos y fáciles de emplear por las poblaciones de la zona rural propensa a estas amenazas.

\section{MATERIALES Y MÉTODOS}

El tipo de investigación de acuerdo a su orientación es Aplicada porque busca la generación de conocimientos con aplicación directa a los problemas de la sociedad o el sector productivo. Se basa en los hallazgos tecnológicos de la investigación básica. También es Experimental, ya que consiste en plantear lo más relevante de un hecho o situación concreta, el diseño es cuasi experimental, pues las variables a evaluar fueron resultados del uso y aplicación de los insumos naturales usados como ayudantes de coagulación para la clarificación del agua. La Población de estudio son las localidades donde existen fuentes superficiales a ser clarificadas especialmente en zonas rurales que no tienen sistemas de agua potable para consumo y cuyas aguas no cumplen con las normas de calidad vigentes. La muestra son las aguas obtenidas de las fuentes superficiales en caseríos y centros poblados de Huaraz y callejón de Huaylas. La muestra a evaluar tuvo un volumen de 36 litros de agua que se usó en cada simulación de los procesos de clarificación de agua. La recolección de datos se hará mediante el test de Jarras, obteniéndose la Dosis óptima, en esta primera etapa se efectuó varias pruebas para determinar la dosis optima del coagulante natural, como son: Tara, cascara de mashua y papa, penca, trigo y semilla de habas agregándole diversas cantidades de los coagulante a cada una de las jarras que contenía agua de muestra. Luego se hizo pruebas para hallar la concentración óptima del coagulante y $\mathrm{pH}$ óptimo con la dosis óptima ya obtenida. Durante el proceso, se determina el índice de willcomb y el tiempo inicial de formación de flóculo en la jarra. Se determinó la turbiedad residual (Tf) a cada muestra, se calculó la eficiencia remocional (Tf/To) y se registran los datos en el graficas Turbiedad (NTU) vs Tiempo (min) y este grafico nos permite obtener tiempo 
de floculación total. Posteriormente se grafica Turbiedad (NTU) vs gradientes de velocidad la cual nos permitió ubicar los puntos de máxima remoción de cada curva de tiempo, obteniéndose las gradientes de velocidad con los que cada tiempo de retención se optimiza. Estos puntos nos permitieron obtener la ecuación de la curva más aproximada, se procede a utilizar el método de mínimos cuadrados. Se determinan los valores de "a "y "b " y el grado de ajuste "R2". Si el grado de ajuste es aceptable (R2 aproxima $=0,90)$, se continua el cálculo, en caso contrario se deberá repetir el procedimiento de laboratorio. Con los valores obtenidos, se determina el gradiente óptimo aplicación la ecuación obtenida que será del tipo: $G^{n}{ }_{\times} T=G^{n} \times T=\mathrm{n}$ y k se determina como consecuencia de este estudio, la cual nos permite obtener la gráfica Gradiente s-1 vs Tiempo de retención (min).

\section{RESULTADOS}

Remoción de la turbiedad: Después de cada ensayo realizado con la dosis, concentración y ph óptimo se analizó el filtrado con papel de filtro para luego llevar al análisis de laboratorio.

Tabla 1. Eficiencias y remoción de turbiedad

\begin{tabular}{|c|c|c|c|c|c|}
\hline coagulante & Muestra & $\begin{array}{c}\text { muestreo } \\
\mathrm{n}^{\circ} 01\end{array}$ & $\begin{array}{c}\text { muestreo } \\
\mathrm{n}^{\circ} 02\end{array}$ & $\begin{array}{c}\text { muestreo } \\
\mathrm{n}^{\circ} 03\end{array}$ & $\begin{array}{c}\text { muestreo } \\
\mathrm{n}^{\circ} 04\end{array}$ \\
\hline \multirow{3}{*}{ MASHUA } & Afluente (NTU) & 9,8 & 43,2 & 36.6 & 46,7 \\
\hline & efluente(NTU) & 8,71 & 5,33 & 2,39 & 5,02 \\
\hline & \% de remoción & $11 \%$ & $88 \%$ & $93 \%$ & $89 \%$ \\
\hline \multirow{3}{*}{ PAPA } & afluente(NTU) & 9,8 & 43,2 & 36,6 & 46,7 \\
\hline & efluente(NTU) & 9,48 & 3,63 & 2,69 & 2,82 \\
\hline & \% de remoción & $3 \%$ & $92 \%$ & $93 \%$ & $94 \%$ \\
\hline \multirow{3}{*}{ TARA } & afluente(NTU) & 31,1 & 52,3 & 47.2 & 25,9 \\
\hline & efluente(NTU) & 1,19 & 1,35 & 2,73 & 2,33 \\
\hline & \% de remoción & 96,17 & 97,42 & 94,22 & 90 \\
\hline \multirow{3}{*}{ TRIGO } & afluente(NTU) & 78,2 & 13,7 & 40,7 & 26,2 \\
\hline & efluente(NTU) & 7,55 & 2,72 & 5,94 & 7,51 \\
\hline & \% de remoción & 90,3 & 80,1 & 85,4 & 71,3 \\
\hline \multirow{3}{*}{ PENCA } & afluente(NTU) & 78,2 & 13,7 & 40,7 & 26,2 \\
\hline & efluente(NTU) & 7,86 & 4,3 & 8,32 & 8,39 \\
\hline & \% de remoción & 89,9 & 68,6 & 79,6 & 67,9 \\
\hline
\end{tabular}


En la tabla 1 se muestra la comparación de las eficiencias de remoción de la turbiedad calculadas en cada coagulante natural, una vez obtenido este dato se puede graficar para tener una mejor visualización de la remoción del material coloidal en las diferentes muestras de agua. Se puede apreciar que la papa presenta eficiencias altas de remoción que llegan a un máximo de 94\% de remoción del material coloidal. Para el caso de Tara que se aplicó a aguas del rio Paria, cuenta con una remoción considerable de material coloidal con una eficiencia máxima de 97\%. Para el caso de la penca y el trigo que se aplicaron a las aguas del río Chucchun, en la cual se verificó en los análisis finales de laboratorio que en el trigo se presenta una eficiencia de $90 \%$ y para la penca la eficiencia llega a un valor del $89 \%$ como máximo.

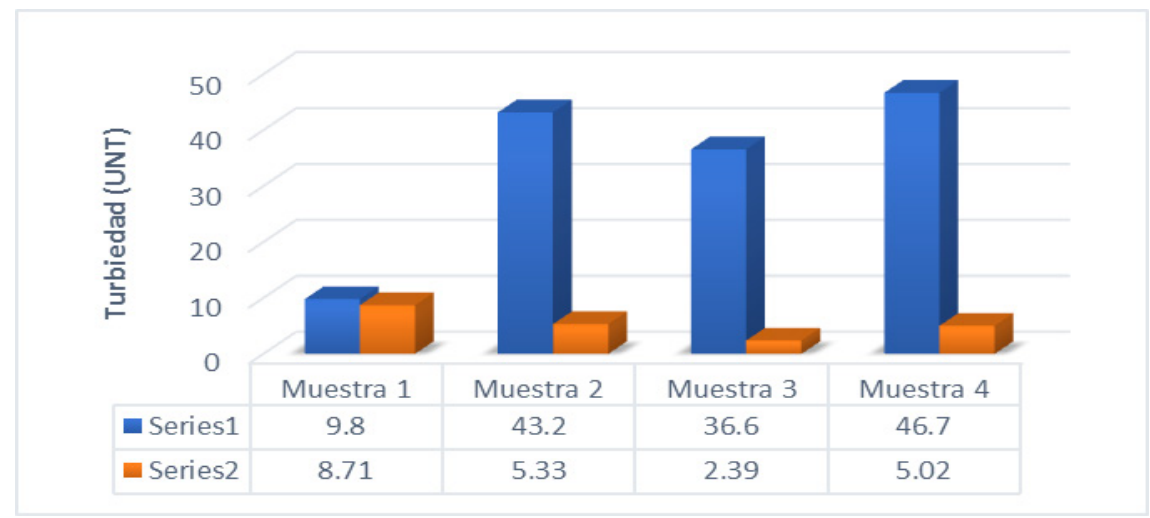

Figura 1. Remoción y turbiedad con mashua

La figura 1 muestra los resultados de las muestras evaluadas con la mashua, obteniéndose en el primer muestreo valores muy cercanos y eficiencias bajas lo cual se puede definir que hubo un error de medición de las muestras, ya que en el 75\% de los monitoreos analizados existe una remoción significativa con valores por debajo a la norma cuyo valor es 5 NTU, frente a valores bajos de 2,39 NTU.

Remoción de sólidos suspendidos totales: Así mismo empleando cada uno de los coagulantes naturales en las aguas superficiales del rio Paria (Huaraz), rio Llullan (Huaylas - Caraz) y el río Chucchun de la provincia de Carhuaz. Se determinó la eficiencia de remoción de los sólidos suspendidos totales, de acuerdo al siguiente cuadro: 
Tabla 2. Eficiencias y remoción de Solidos Suspendidos Totales

\begin{tabular}{cccccc}
\hline \multirow{2}{*}{ coagulante } & & $\begin{array}{c}\text { muestreo } \\
\mathrm{n}^{\circ} 01\end{array}$ & $\begin{array}{c}\text { muestreo } \\
\mathrm{n}^{\circ} 02\end{array}$ & $\begin{array}{c}\text { muestreo } \\
\mathrm{n}^{\circ} 03\end{array}$ & $\begin{array}{c}\text { muestreo } \\
\mathrm{n}^{\circ} 04\end{array}$ \\
\hline \multirow{3}{*}{ MASHUA } & Afluente (mg/L) & 42 & 48 & 115 & 40 \\
& efluente(mg/L) & 67 & 59 & 63 & 53 \\
& \% de remoción & $0 \%$ & $0 \%$ & $45 \%$ & $0 \%$ \\
PAPA & afluente(mg/L) & 42 & 48 & 115 & 40 \\
& efluente(mg/L) & 91 & 54 & 86 & 43 \\
& \% de remoción & $0 \%$ & $0 \%$ & $25 \%$ & $0 \%$ \\
TARA & afluente(mg/L) & 212 & 231 & 232 & 333 \\
& efluente(mg/L) & 64 & 63 & 24 & 80 \\
& \% de remoción & 69,81 & 72,73 & 89,66 & 75,98 \\
TRIGO & afluente(mg/L) & 58 & 66 & 74 & 65 \\
& efluente(mg/L) & 72 & 75 & 64 & 61 \\
& \% de remoción & 0 & 0 & 13,51 & 6,15 \\
PENCA & afluente(mg/L) & 58 & 66 & 74 & 65 \\
& efluente(mg/L) & 62 & 63 & 60 & 71 \\
& \% de remoción & 0 & 4,55 & 18,92 & 0 \\
\hline
\end{tabular}

La tabla 2 muestra las eficiencias de remoción de los Sólidos Suspendidos Totales, en la cual las cáscaras de mashua y de papa muestran variaciones muy dispersas de la capacidad y eficiencia de remoción respecto a los materiales sólidos, presentando la mashua una eficiencia de $45 \%$ y la papa una eficiencia de $25 \%$.

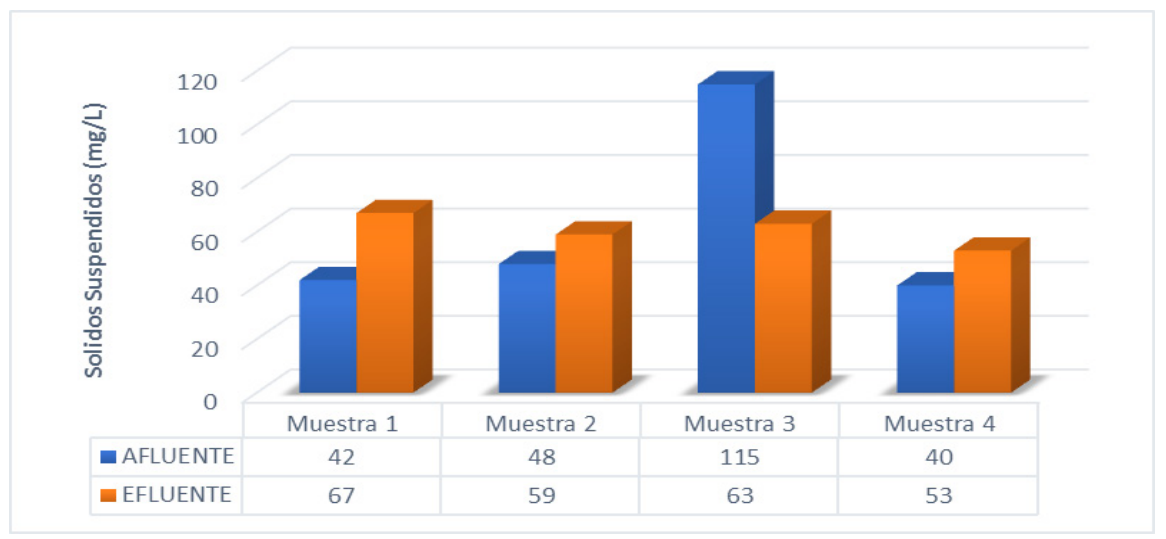

Figura 2. Remoción de sólidos suspendidos con Mashua

En la figura 2 se muestra la capacidad de remoción de sólidos suspendidos totales con el insumo de mashua empleado en las aguas del rio Paria, en la cual se puede apreciar las remociones en los diversos muestreos, en donde se obtiene una eficiencia máxima de $45 \%$ y se comprueba que si tiene la capacidad de remover esos contaminantes presentes en agua superficial en los ensayos presentados. 


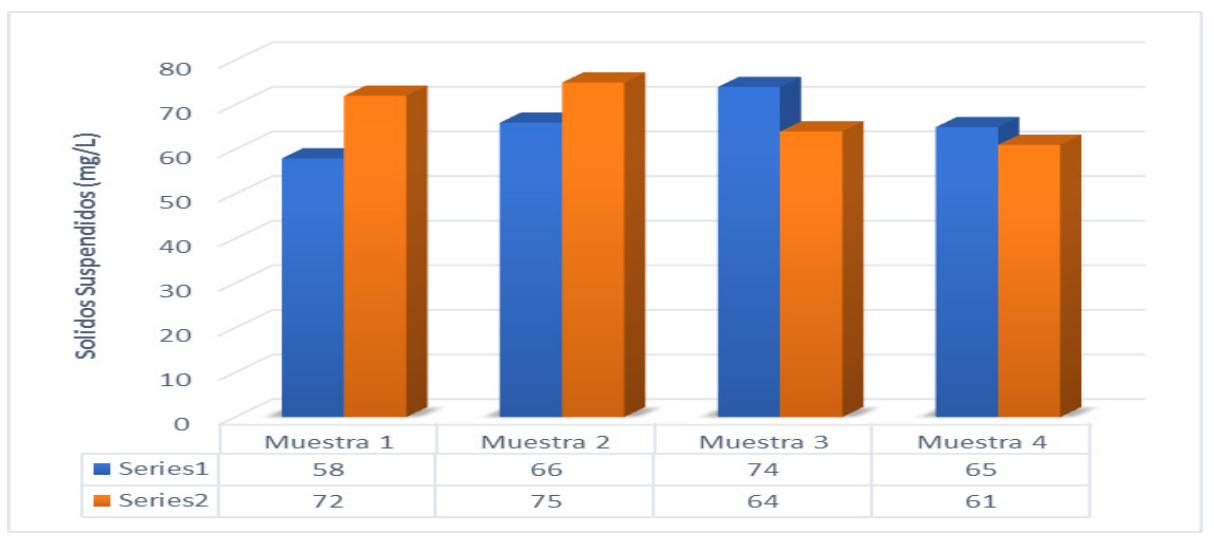

Figura 3. Remoción de sólidos suspendidos totales con Trigo

En la figura 3 se muestra la capacidad de remoción de sólidos suspendidos totales con el insumo de trigo que con los ensayos se demostró que la eficiencia del tratamiento realizado no se obtuvo porcentajes altos de tratabilidad, mostrando un valor máximo de $13,51 \%$.

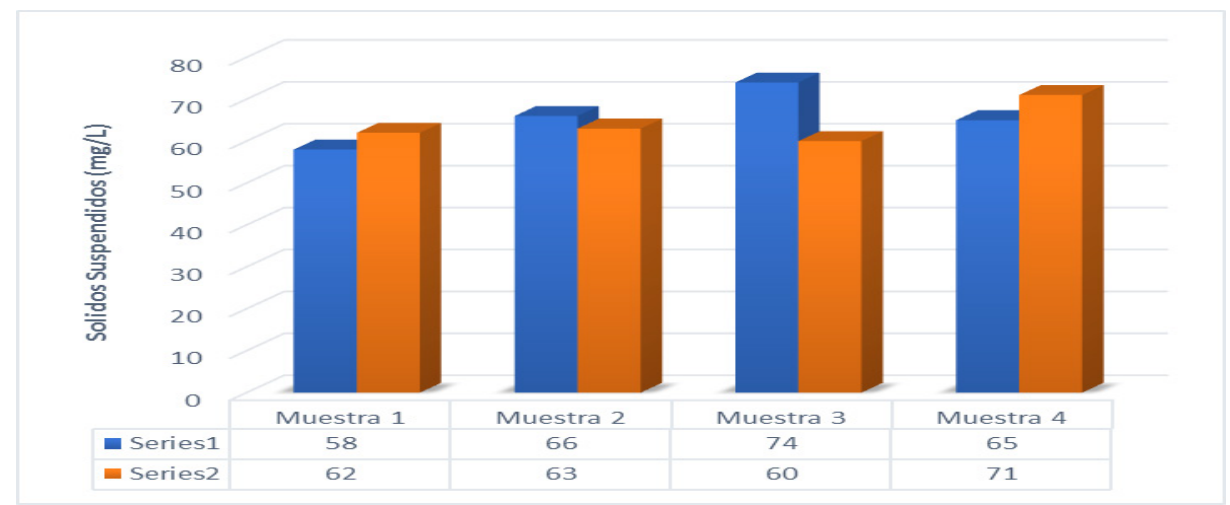

Figura 4. Remoción de sólidos suspendidos totales con Penca

En la figura 4 se muestra la capacidad de remoción de sólidos suspendidos con el insumo de penca que con los ensayos se demostró que la eficiencia del tratamiento realizado presenta porcentajes altos de remoción llegando a un valor máximo de 18,92\% y valores bajos de 4,55\%, lo cual indica que la cantidad de sólidos suspendidos no son removidos de manera considerable. 


\section{DISCUSIÓN}

El propósito del presente trabajo es evaluar si es efectivo el uso de especies naturales como ayudantes de coagulación para la clarificación de aguas turbias en épocas de avenidas en la zona se Huaraz y el Callejón de Huaylas. Se realizó el análisis de calidad de agua a las muestras tomadas donde se aplicó el coagulante natural y se determinó por el método de validación de hipótesis " $\mathrm{t}$ ” que todos los insumos: papa, mashua, tara, trigo y penca remueven efectivamente la turbiedad a una confiabilidad del $90 \%$. Así mismo se comprobó que cuando se realizó la validación de la hipótesis para cada coagulante se tuvo que aceptar la hipótesis nula para los sólidos disueltos totales, pues no se apreció una remoción efectiva estadísticamente. Se evaluó las causas de la no eficacia en uno de los indicadores, pues esta se originó por no realizar un análisis adicional y obtener otras concentraciones y dosis para la remoción de solidos disueltos.

Por otra parte, Pompilio (2013) sostiene que la extracción del coagulante de la especie Opuntia, lo cual se realizó utilizando acetona como solvente, lográndose así un rendimiento entre 2,5 y 7,0 \% en base húmeda para remover la turbidez del agua en agua del Río Santa. Los resultados que se obtuvieron en la aplicación como coagulante y coadyuvante se reflejaron en la formación de flocs, dando a éstos formación casi inmediata y con buenas características (en tamaño principalmente), aun cuando en la sedimentación se presentaron algunas variaciones, las cuales se logran confirmar, con las pruebas físicas realizadas al sobrenadante, estas para las muestras con color y de agua clarificada luego de la sedimentación. De acuerdo a los resultados obtenidos en la remoción de turbidez en las muestras de agua del Rio Santa, con el uso solamente de floculantes naturales y sin sulfato de aluminio, la remoción que presentó no es la esperada debido a una sedimentación deficiente, ya que con el coadyuvante se aumenta el tamaño del floc pero no se consistencia e incremento de peso. En los resultados que se presentan al aplicar el coagulante natural en agua turbia del Río Santa se muestra mejor eficiencia utilizando Opuntia ficcus con un porcentaje de remoción de turbidez total de 75,4\%.

En la ciudad de Huaraz en la fuente del rio Llullan se obtuvo la turbiedad de 34,0 NTU, y se determinó una dosis óptima, según los resultados la turbiedad más baja se observa en la dosis de $37 \mathrm{mg} / 1$ por lo tanto viene a ser la dosis óptima con una concentración de $4 \%$ por lo que se considera como la concentración óptima. Y se observó que al agregar el coagulante de cascara de papa a una concentración de $4 \%$ en un pH de 8 los flocs empezaron a formarse por ende a sedimentar después de la prueba, obteniendo la turbiedad más baja en el tratamiento.

Por otra parte, Aguilar (2010) sostienen que la utilización de polímeros naturales es una interesante alternativa por su bajo costo en comparación con los polímeros sintéticos, en el caso de este producto es totalmente natural e inocuo para las personas. La goma de tara se produce en el Perú en grandes cantidades para la exportación al extranjero para su uso en el sector alimentario y diversas aplicaciones en la industria, es por ello que se debe estudiar su factibilidad en el tratamiento de aguas. Se realizó pruebas de jarras con aguas sintéticas preparadas en el laboratorio, la primera muestra denominada agua tipo I con una turbiedad de 200 NTU y la segunda agua mucha más claras con una turbiedad de 25 NTU. A los dos tipos de agua se les realizaron pruebas utilizando el sulfato de aluminio solo como coagulante primario y en una segunda fase usando 
el sulfato de aluminio como coagulante primario y agregando la goma de tara como polímero natural para mejorar su rendimiento. En este trabajo se hizo un análisis de la eficiencia de la goma de tara, ya que esta no solo permite la formación de mejores flocs y por ende una mejor sedimentación, sino también permite arrojar sulfato de aluminio que es el coagulante más utilizado en el tratamiento de aguas.

Para la evaluación del coagulante del trigo, los resultados la turbiedad más baja es de 23,4NTU con una dosis optima de $45 \mathrm{mg} / \mathrm{L}$, por lo tanto, viene a ser nuestra de la dosis con buena eficiencia de remoción con la turbiedad más baja tiene una concentración de $5,3 \%$ por ende viene a ser nuestra concentración óptima para dicho muestreo. A una concentración de 5,3\% en un $\mathrm{pH}$ de 8 los flocs empezaron a formarse por ende a sedimentar después de la prueba, obteniendo la turbiedad más baja en el tratamiento. Además, para la evaluación del coagulante natural la penca, según los resultados la turbiedad más baja es de 16,0 NTU con una dosis optima de $36 \mathrm{mg} / 1$, por lo tanto, viene a ser nuestra de la dosis con buena eficiencia de remoción con la turbiedad más baja 15,8 NTU tiene una concentración de $4,5 \%$ por ende viene a ser la concentración óptima para el coagulante natural aplicado. Se observó que al agregar el coagulante de penca a una concentración de 4,5\% en un $\mathrm{pH}$ de 7,5 los flocs empezaron a formarse por ende a sedimentar después de la prueba, obteniendo la turbiedad más baja en el tratamiento. De acuerdo al CEPIS (2004) indican que para que los procesos de potabilización de agua empleando insumos químicos coagulantes el $\mathrm{pH}$ debe estar en un valor de 6,5 a 8,5 , en la investigación se demostró que con los coagulantes y polímeros naturales se logró obtener valores de $\mathrm{pH}$ en promedio de 7,0 a 7,9.

\section{CONCLUSIONES}

El uso de especies naturales como ayudantes de coagulación para la clarificación de aguas turbias en los centros poblados de Huaraz y el Callejón de Huaylas, son eficientes de acuerdo a los indicadores evaluados como turbiedad y sólidos disueltos que son parámetros típicos y característicos de aguas superficiales.

La calidad del agua se obtuvo de las cuencas del rio Llullan (Caraz), Paria (Huaraz) y Chuchun (Carhuaz); que son fuentes de abastecimiento de agua para más de 50 habilitaciones urbanas y rurales, entre ellas la Ciudad de Huaraz y Carhuaz, que de acuerdo al análisis se comprobó un nivel de turbiedad de 46,7 NTU para rio Llullan, Paria de 52,3 NTU y Chucchun con 78,2 NTU.

Una vez identificado los fuentes de agua y los coagulantes a emplear se determinó la dosis óptima para la mashua fue de $28 \mathrm{mg} / \mathrm{L}$ a una concentración de 3,5\% y con un pH de 8,0; parael caso de la papa se comprobó que la dosis empleada fue de $37 \mathrm{mg} / \mathrm{L}$ a una concentración de \% con un $\mathrm{pH}$ de 8,0; para el coagulante de tara se hizo los análisis con una valor de dosis de $35 \mathrm{mg} / \mathrm{L}$ con una concentración de 3,2\% a un pH de 7,5; para el coagulante de trigo se empleó una dosis optima de 45,0 $\mathrm{mg} / \mathrm{L}$ a una concentración de $5,3 \%$ con un $\mathrm{pH}$ de la fuente de agua a 8,0 ; y para el caso de la penca la dosis empleada fue de $36 \mathrm{mg} / \mathrm{L}$ a una concentración de 4,5\% y un $\mathrm{pH}$ de 7,5. 
Una vez empleado el coagulante en la muestra de agua se sometió al análisis final de caracterización y en donde se aprecia una eficiencia en la turbiedad aceptable de acuerdo a la normatividad de calidad de agua para consumo humano, logrando valores para la mashua 93\% de remoción de turbiedad, para la papa de 94\%, cuando se empleó la tara en el rio Paria alcanzo un remoción de 97\% y mientras que las aguas del rio Chucchun donde se sometió al trigo logro una eficiencia de $90 \%$ y el alcanzado por la penca es de 89,9\% obteniendo valores por debajo de 5,0 NTU.

Para el caso de los sólidos disueltos totales se logró pocos rangos de remoción para la mashua se logró un 45\%, para la papa 25\%, tara logro una remoción más alta al 89\% en un solo ensayo; el trigo llego a una eficiencia de 13,5\% mientras que el penca logro una remoción de 18,9\% siendo validado por los datos mostrados en los resultados.

\section{REFERENCIAS BIBLIOGRÁFICAS}

CEPIS. 2004. «Tratamiento de agua para consumo humano Plantas de filtración rápida. Manual I». Lima - Organización Panamericana de la Salud.

Aguilar, Edwar. 2010. «Utilización de las Semillas de Tara (Caesalpinia Spinosa) como Ayudante de Coagulación en el Tratamiento de Aguas». Lima.

Pompilio, Carlos. 2013. «Uso de Floculantes de Origen Natural en el Tratamiento del Agua en Términos de Turbidez en el Río Santa - Huaraz». Ancash.

Kumar, M. 2015. «Coagulation performance evaluation of natural and synthetic coagulants in waste water treatment». <http://www.arpnjournals.com/jeas/research_papers/rp_2015/jeas_0415_1820.pdf> [Consulta: 23-08-2017].

Rodiño, Johana. 2015. «Sinú River raw water treatment by natural coagulants».<http: / / www.scielo.org.co/scielo.php?script=sci_arttext\&pi$\mathrm{d}=$ S0120-62302015000300011> [Consulta: 08-08-2017].

Pavanakumar, Pastay; Venkatareddy; Koppad and Keerthi, Gowda. 2017. «Application of Natural Coagulants in Removal of Turbidity and Hardness». <https:// www.researchgate.net/publication/315396691_Application_of_Natural_Coagulants_in_Removal_of_Turbidity_and_Hardness> [Consulta: 23-08-2017].

Yunusa, U. 2017. «Cucurbita pepo Seed Powder Reduce the Turbidity of River Water». $<$ https://www.scholarsresearchlibrary.com/articles/cucurbita-pepo-seed-powder-reduce-the-turbidity-of-river-water.pdf $>$ [Consulta: 23-08-2017].

Recepción: 14/04/2018

Aceptación: 01/11/2018

\section{Correspondencia}

Dávila Paredes Cesar Manuel

cemagre@hotmail.com 